PROGRAMA DE INICIAÇÃO CIENTÍFICA

VINÍCIUS BORGES VIEIRA

RESPOSTAS AGUDAS DE TESTOSTERONA E CORTISOL EM MULHERES NÃO TREINADAS AO EXERCÍCIO EXCÊNTRICO 


\section{Unce $=0$ \\ Centro Universitb́no de Brasilıa}

\section{VINÍCIUS BORGES VIEIRA}

\section{RESPOSTAS AGUDAS DE TESTOSTERONA E CORTISOL EM MULHERES NÃO TREINADAS AO EXERCÍCIO EXCÊNTRICO}

Relatório final de pesquisa de Iniciação Científica apresentado à Assessoria de Pós-Graduação e Pesquisa pela Faculdade de Ciências da Educação e da Saúde - FACES.

Orientação: Alessandro de Oliveira Silva. 


\title{
RESPOSTAS AGUDAS DE TESTOSTERONA E CORTISOL EM MULHERES NÃO TREINADAS AO EXERCÍCIO EXCÊNTRICO
}

\author{
Vinícius Borges Vieira - UniCEUB, PIC Institucional, aluno bolsista \\ vini9000@hotmail.com.com
}

\begin{abstract}
Alessandro de Oliveira Silva - UniCEUB, professor orientador silva.alessandro.oliveira@gmail.com
\end{abstract}

Mateus Medeiros Leite - UniCEUB, colaborador

mateus.edf@outlook.com

\section{Silvana SchwerzFunghetto - UnB, professor colaborador silvana.funghetto@gmail.com}

A reposta de Testosterona e Cortisol ao Treinamento de Força tem sido usada na tentativa de marcar o estado anabólico-catabólico do corpo na qual a Testosterona um hormônio sexual masculino com importante contribuição no controle metabólico e de característica Anabólica, e o Cortisol, um hormônio com característica Catabólica, na qual seu aumento de longa duração tem sido mostrado para exibir um efeito de inibição sobre o sistema neuromuscular. O objetivo do presente estudo foi verificar os níveis circulantes hormonais de testosterona e cortisol em repouso e após realização de um determinado protocolo de exercícios excêntricos em mulheres não praticantes de exercícios físicos. Tratou-se de um estudo transversal realizado com 10 mulheres (idade: $23,10 \pm 3,75)$ não praticantes de exercícios físicos. As voluntárias foram avaliadas antropometricamente por meio de bioimpedância (IMC: 23,97 $\pm 3,49$; \% gordura: 32,40 $\pm 5,58$; massa muscular esquelética: $22,21 \pm 2,54)$. Foram submetidas à sessão de adaptação ao exercício proposto, determinação da carga de 10 repetições máximas (10 RM: 62,42 + 12,48) e a sessão de exercício, que foi realizado na cadeira extensora de forma bilateral com $110 \%$ da carga máxima obtida no teste de 10 RM, composta de 7 séries com 10 repetições. As análises das respostas hormonais foram realizadas por coletas sanguíneas pré-exercício e logo após execução do exercício. Os valores adotados para diferenças estatisticamente significativas através do Teste t pareado foi de $(p \leq$ 0,05).As concentrações de Cortisol nas condições pré-intervenção e pós-exercício foram significativamente diferentes, com aumentos nos níveis séricos logo após execução do exercício (pré: 5,65 $\pm 2,00 \mathrm{mcg} / \mathrm{dL}$; pós: $7,35 \pm 2,76 \mathrm{mcg} / \mathrm{dL}$; $\mathrm{p}=$ $0,0017)$. A concentração de Testosterona também indicou diferenças estatisticamente significativas, com um decréscimo nos valores pós exercício (pré: $43,40 \pm 23,16 \mathrm{ng} / \mathrm{dL}$; pós: $38,60 \pm 18,93 \mathrm{ng} / \mathrm{dL} ; \mathrm{p}=0,01)$. Portanto, observou-se a predominância de um estado catabólico pós a realização de uma sessão de treinamento de força excêntrico, o qual induziu respostas agudas com maiores níveis de cortisol e menores níveis de testosterona comparados aos níveis em repouso. É de grande importância a realização de futuras pesquisas visando analisar os níveis destes hormônios em um estado agudo tardio e também a análise destes de forma crônica.

Palavras-chave: Respostas Hormonais. Treinamento de Força. Mulheres. 


\section{LISTA DE ABREVIATURAS}

(T:C)- Razão Testosterona: Cortisol

(10 RM)- Teste de 10 Repetições Máximas.

(TFE)- Treinamento de força excêntrico 


\section{INTRODUÇÃO}

A Testosterona é considerada um hormônio que tem relação com a Síntese e diminuição da degradação de proteína muscular. É um hormônio masculino de função anabólica e androgênica, produzido pelas células de Leydig nos testículos. Há também uma pequena quantidade de secretada pelas glândulas supra-renais (ARAÚJO, 2008).

Em mulheres, segundo Crewther et al., (2006) apud Gerlinger-Romero et al., (2014) os ovários e adrenais são os responsáveis pela produção da testosterona. A Testosterona é mais produzida e liberada por indivíduos do sexo masculino, o que faz jus a sua função, maior massa muscular e características como mais pêlos, voz mais grave, produção de espermatozóides, etc. A testosterona também está presente no sistema feminino em menores quantidades, e depende da biossíntese de glicocorticóide, na qual o córtex adrenal, secreta esteróides androgênicas que podem ser convertidos em testosterona na periferia (GERLINGER-ROMERO et al., 2014).

O cortisol é um hormônio de função catabólica, é um dos principais glucocorticóides em seres humanos sendo secretado a partir do córtex adrenal em resposta ao estresse físico e/ou psicológico Em resposta a esse estresse, o eixo Hipotálamo-Pituitária-Adrenal (HPA) é ativado e, conseqüentemente, ocorre a liberação de cortisol, que portanto exerce um papel importante no equilíbrio eletrolítico e no metabolismo de carboidratos, proteínas e lipídeos (BROWNLEE et al., 2005; MORAES et al., 2012; GUYTON, 1998).

A função catabólica do Cortisol está ligada ao metabolismo de carboidratos, proteínas e lipídeos, e sua ação por níveis elevados, se prolongada, pode levar à proteólise muscular e perda de massa. O cortisol estimula o fracionamento das proteínas e acelera a utilização das gorduras para obtenção de energia, através da lipólise (SIMMONS et al., 1984; FRANÇA et al., 2006).

Como o Cortisol e poucas quantidades de Testosterona ambos são secretados a partir do córtex adrenal, sugere que em reposta à um estimulo físico ou psicologico, a glândula suprarenal pode produzir o cortisol, é há a possibilidade que alguma Testosterona livre seja produzida e secretada em simultâneo, levando ao 
aumento das concentrações circulantes de ambas os hormônios (BROWNLEE et al., 2005).

A reposta destes dois hormônios, relação Testosterona: Cortisol ao exercício físico, sobretudo Treinamento de Força, tem sido usada na tentativa de marcar o estado anabólico-catabólico do corpo, sendo a Testosterona, hormônio sexual masculino com importante contribuição no controle metabólico e de característica Anabólica, e o Cortisol, um hormônio Catabólico na qual seu aumento de longa duração tem sido mostrado para exibir um efeito de inibição sobre o sistema neuromuscular (COLTINHO et al., 2007; TEO et al., 2011).

Muitos estudos que relacionam a resposta hormonal da Testosterona e Cortisol com o exercício físico estudam esta resposta Aguda e/ou Crônica, sendo a população basicamente já adaptada a algum método de treinamento, onde na maioria das vezes os indivíduos investigados são homens e já praticantes de algum exercício, sobretudo, treinamento de força. A partir do que propõe alguns estudos, os níveis de Cortisol são aumentados pós exercício, havendo uma resposta negativa da razão T:C, prevalecendo o estado Catabólico, como proposto pelo estudo de (UCHIDA et al., 2006).

Diante disto, a relevância deste trabalho foi em, diferentemente da maioria dos estudos com a mesma linha de pesquisa, criar um estudo que verificasse as respostas hormonais Agudas de Testosterona e Cortisol circulante, em níveis basais e pós-execução de um determinado protocolo de Exercício Excêntrico para o grupo de mulheres. Portanto o Objetivo do presente estudo foi verificar os níveis circulantes hormonais de testosterona e cortisol em repouso e após realização de um determinado protocolo de exercícios excêntricos em mulheres não praticantes de exercícios físicos.

\section{FUNDAMENTAÇÃO TEÓRICA}

O treino de força é conhecido como forte estimulador para respostas do sistema endócrino. O exercício excêntrico é conhecido por ser um método de treinamento eficiente em relação ao aumento de força e hipertrofia muscular e a sua eficácia é possivelmente mediada pela capacidade de atingir forças mais elevadas durante as ações musculares excêntricas. A manipulação da velocidade excêntrica 
parece ser um fator de influências metabólicas, os músculos permanecem sob tensão na realização do exercício, o que conseqüentemente poderá afetar as respostas metabólicas agudas. (KRAEMER; RATAMESS 2005; ROING et al., 2008; CALIXTO et al., 2014).

Os fatores que possivelmente influenciam as respostas hormonais para mensuração de níveis de Testosterona e Cortisol, correlacionam esta de forma aguda a variáveis ligadas a sessão de treino, como, numero de series e repetições, carga e tempo de intervalo, o que confere uma plasticidade na resposta do sistema endócrino ao exercício. A identificação de tais fatores estimulantes de respostas hormonais se torna importante para que haja uma melhor periodização e prescrição de treinamento proporcionando um ambiente metabólico em relação a determinados objetivos (CADORE et al., 2008; BOTH, 2010)

Para que haja adaptações fisiológicas em resposta ao treino de força, o músculo deve ser submetido a uma carga de esforço acima daquela que habitualmente está acostumado a suportar, sendo um treino de volume com alta intensidade a melhor estratégia para aumentar os níveis de testosterona e talvez diminuir os níveis de cortisol. A testosterona parece aumentar após sessões curtas e intensas de treinamento, principalmente de força, já o cortisol parece aumentar com sessões longas e intensas de treinamento, principalmente aeróbio (SÁ et al., 2014; ARAÚJO, 2008)

O Exercício físico em relação ao Cortisol está relacionado com a intensidade do esforço realizado, em que os esforços mais intensos tendem a induzir maiores concentrações devido a elevação demanda metabólica e a sua importância em relação ao exercício físico se dá por seus efeitos catabólicos exercidos sobre o músculo esquelético (SILVA et al. 2014; RAHIMI et al., 2011).

Alterações na concentração destes hormônios estão associadas na modulação de diversas respostas induzidas pelo treinamento, como hipertrofia e ganho de força, o que se pode dar pela razão $\mathrm{T}: \mathrm{C}$, de determinação de estado Anabólico ou Catabólico. Estudos propõem haver uma relação negativa de (T:C) aguda, após execução do exercício, porém, esta relação inverte-se na fase de recuperação, o que possivelmente poderia se associar que a resposta crônica seria positiva de promoção de ambiente Anabólico (UCHIDA et al., 2004). 
Sabendo que a relação entre os níveis de Testosterona e Cortisol se utiliza a fim de verificar o índice de esforço na pratica de exercícios físicos, e grande parte dos estudos terem suas amostras compostas por homens com experiência em algum exercício físico, resta saber as repostas destes hormônios em mulheres destreinadas.

\section{METODOLOGIA}

A presente pesquisa teve caráter experimental e natureza quantitativa, realizada com mulheres não praticantes de exercícios físicos.

\section{Local de realização da pesquisa:}

As medidas de composição corporal foram realizadas nos Labociens do Uniceub. A fase de adaptação ao exercício, teste de 10 RM e aplicação do treino excêntrico juntamente com as coletas sanguíneas foram realizadas na Academia Escola no Centro de Atendimento Comunitário do UniCEUB.

\section{Amostra:}

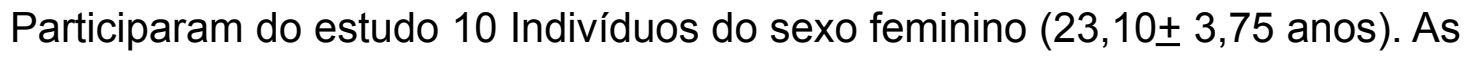
voluntárias foram recrutadas através da divulgação da pesquisa internamente no UniCEUB, as quais atenderam aos seguintes critérios de inclusão: ser sedentária ou não praticar atividade física nos últimos 12 meses, não possuíssem problemas ortopédicos e articulares que impediam a realização da atividade física excêntrica ou que pudesse agravar o dano osteoarticular, e que concordassem em assinar o termo de consentimento livre e esclarecido. Foram excluídas aquelas que alegaram ter praticado exercícios físicos regularmente nos últimos 12 meses anteriores a pesquisa, que possuíam alguns problemas ortopédicos e articulares nos membros inferiores e que não assinaram o termo de consentimento livre e esclarecido. Este projeto foi submetido e aprovado sobe CAAE: 15900813.1.0000.0023

\section{Instrumentos e procedimentos metodológicos:}

Os procedimentos para caracterização da amostra, sobretudo a composição corporal, foi realizada por bioimpedância (INBODY 570), e o programa de TFE, em todas suas fases, foi realizado $\mathrm{n}$ cadeira extensora de forma bilateral. As coletas 
sanguíneas foram realizadas por enfermeira devidamente treinada nos momentos pré e logo após a execução do protocolo de TFE.

\section{Avaliação da composição corporal:}

As medidas de composição corporal foram realizadas por bioimpedância (INBODY 570), que larga uma corrente elétrica alternada segura (Imperceptível ao ser humano) no corpo do sujeito, entre o braço e perna do mesmo lado da pessoa, através dessa corrente elétrica são medidos valores de bioimpedância, que seria uma resistência natural a passagem de corrente elétrica. Para os resultados obtidos foram analisados: Composição corporal, Músculo- gordura, Análise de Obesidade (IMC e \% de gordura), Massa magra, Circunferência da Cintura (CC), Circunferência do quadril $(C Q)$ e sua relação $R C Q$ (relação cintura-quadril). Para todas estes medidas, será pedido aos participantes que estejam com o mínimo de roupa possível. Medida de estatura será realizada com estadiômetro de parede (Sanny, São Paulo, Brasil), com capacidade de 2200 mm e divisão de1 mm.

\section{Análise da Testosterona e Cortisol:}

As coletas sanguíneas para definição dos níveis de Testosterona e Cortisol foram realizadas por punção venosa de uma veia do antebraço, este procedimento foi realizado por uma enfermeira devidamente treinada. As coletas se deram no momento pré exercício, anteriormente ao aquecimento e logo após a conclusão da última série do exercício, em ambiente amplo e arejado.

Procedimentos experimentais de Atividade Física: antes de iniciar a execução do programa de exercício de força excêntrico, as voluntárias foram submetidas à fase de adaptação ao exercício e avaliação da força máxima, seguindo os procedimentos abaixo descritos:

- Fase de adaptação ao exercício: Por se tratar de uma população não praticante de exercícios regulares, as voluntárias foram submetidas a uma fase de adaptação ao exercício, para conhecerem de qual exercício se tratava e quais padrões adotados para a execução do mesmo.

- Avaliação de Força Máxima: O teste de 10 RM foi realizado na cadeira extensora de forma bilateral, utilizado para avaliar a força máxima. Primeiramente, as participantes realizaram o aquecimento geral com duração 
de 10 minutos em esteira em intensidade leve; posteriormente, foram submetidas a oito repetições com 50\% de carga de 10RM. A carga foi estimada de acordo com a capacidade de cada participante verificada na sessão de adaptação realizada nos dias anteriores. Após um minuto de intervalo, foram realizadas três repetições com $70 \%$ de $10 \mathrm{RM}$ estimada. Depois de três minutos, as tentativas subsequentes foram realizadas com cargas progressivamente mais pesadas, até a determinação da carga de 10RM, com um máximo de três tentativas, entre três a cinco minutos de intervalo entre cada tentativa. As padronizações das angulações e movimentos dos exercícios foram conduzidas de acordo com as descrições de Funghetto, et al. (2014).

- Aplicação do treino excêntrico: Após dois dias de descanso as voluntárias foram submetidas a uma série de aquecimento geral em cicloergômetro de membros inferiores durante 10 minutos a $60 \mathrm{rpm}$ e $50 \mathrm{w}$,seguido de aquecimento especifico de 10 repetições a $50 \%$ de $1 \mathrm{RM}$, com intervalo de 3 minutos no mesmo horário em que fizeram o treino de avaliação. A sessão de treino na cadeira extensora bilateral foi realizada $120 \%$ de $1 \mathrm{RM}$, somente na fase excêntrica, sendo a fase concêntrica executada pelo avaliador. Foram utilizadas sete séries de 10 repetições com descanso passivo de 3 minutos entre as séries e com intervalos de $15 \mathrm{~s}$ entre cada repetição de 2 segundos. As participantes do estudo foram orientadas a realizar a extensão dos joelhos mesmo que não consigam vencer a resistência.

\section{Análise Estatística}

Inicialmente os dados foram tratados a partir dos procedimentos descritivos, com as informações sendo processadas no pacote computacional BioEstat em sua versão 5.3. Medidas de tendência central e variabilidade dos dados foramrepresentadas como média e desvio padrão. Foi utilizado coeficiente de correlação de Spearman e teste t pareado para comparação dos valores pré e pósexercício. O nível de significância adotado foi de $(P \leq 0,05)$. 


\section{RESULTADOS E DISCUSSÃO}

Os dados de caracterização da amostra quanto à Idade, Força obtida no teste de 10 RM, e Composição Corporal geral realizada por meio de bioimpedância, estão indicados na Tabela 1 com média e desvio padrão.

Tabela 1. Valores antropométricos e caracterização da amostra.

\begin{tabular}{cc}
\hline VARIÁVEIS & VALORES \\
\hline Idade (anos) & $23,10 \pm 3,75$ \\
\hline MC (kg) & $60,52 \pm 9,25$ \\
\hline 10 RM (kg) & $63,40 \pm 13,09$ \\
IMC & $23,97 \pm 3,49$ \\
\% & $32,40 \pm 5,58$ \\
MME & $22,21 \pm 2,54$ \\
MLG & $40,59 \pm 4,15$ \\
\hline Massa Corporal. 10 RM= 10 repetições máximas. IMC= Índice de Massa \\
ral. \%G= Percentual de Gordura. MME= Massa Muscular Esquelética. MLG= \\
\end{tabular}

Tabela 2. Valores hormonais da amostra, pré e pós-exercício.

\begin{tabular}{ccc}
\hline Variável & Pré Exercício & Pós Exercício \\
\hline Testosterona & $43,41 \pm 23,17(\mathrm{ng} / \mathrm{dL})^{*}$ & $38,60 \pm 18,94(\mathrm{ng} / \mathrm{dL})^{*}$ \\
Cortisol & $5,65 \pm 2,00(\mathrm{mcg} / \mathrm{dL})^{*}$ & $7,35 \pm 2,76(\mathrm{mcg} / \mathrm{dL})^{*}$ \\
\hline & \\
\hline
\end{tabular}

De acordo com os dados apresentados na Tabela 2, foi demonstrado que as concentrações de Cortisol nas condições pré-intervenção e pós-exercício foram significativamente diferentes, com aumentos nos níveis séricos logo após execução do exercício (pré: $5,65 \pm 2,00 \mathrm{mcg} / \mathrm{dL}$; pós: $7,35 \pm 2,76 \mathrm{mcg} / \mathrm{dL} ; p=0,0017$ ). A concentração de Testosterona também indicou diferenças estatisticamente significativas, com um decréscimo nos valores pós exercício (pré: 43,40 $\pm 23,16 \mathrm{ng}$ / dL; pós: $38,60 \pm 18,93 \mathrm{ng} / \mathrm{dL} ; \mathrm{p}=0,01)$. 
Segundo Uchida et al. (2004), foi observado que no período de 8 semanas de treino, a secreção de cortisol antes da sessão de treino foi reduzida em $38 \%$, enquanto após a sessão, essa concentração foi elevada em $48 \%$ em relação ao repouso. Segundo Badillo (2001), em pessoas estressadas ou muito ansiosas a produção de cortisol durante exercício é muito maior, com isso, os indivíduos possuem uma maior dificuldade para se recuperam após o esforço devido ao fato de que possuem uma menor produção de testosterona, com isso, ele afirma que a produção de testosterona é inibida devido a concentração elevada de cortisol no sangue.

Ainda segundo Uchida et al. (2004), a elevação nos níveis de cortisol e a diminuição dos níveis de testosterona são indicadores de sobrecarga de esforço mas não necessariamente de Overtraining, ou seja, de acordo com os resultados na presente pesquisa, os valores obtidos estão relacionados ao esforço exigido durante o treino. De acordo com Viru e Viru (2001), essa alteração nos níveis de cortisol e testosterona são indicativos de overreaching.

Segundo Smilios et al. (2003), em seu estudo, feito com onze jovens homens, diferentes quantidades de trabalho são fatores que afetam a resposta hormonal aguda, dessa forma, foi possível observar que quantidades apropriadas de trabalho criam um ambiente favorável para o desenvolvimento de força e massa muscular, além de criarem uma harmonia entre hormônios catabólicos e anabólicos.

Segundo o estudo de Ahtiainen et al., (2005), realizado com 2 protocolos com $10 \mathrm{RM}$ em homens treinados, aonde o tempo de intervalo era de 2 e 5 minutos, demonstrou que não há diferenças nas respostas hormonais agudas. Porém em atividades aonde a predominância é do sistema glicolítico lático, parecem apresentar um maior estimulo de hormônios esteroides a testosterona.

Salehi et al., (2013) realizaram estudo com mulheres obesas com intuito de descobrirem possíveis mudanças no padrão hormonal de testosterona e cortisol após 8 semanas de prática de um programa de corrida. Foi demonstrado que houve decréscimo significante nos níveis de cortisol após o programa de exercício. Foi observado que não houve alterações significantes nos níveis de testosterona entre o grupo experimental e controle após o término do protocolo de exercício aeróbico, indicando que o exercício aeróbico não foi estressante para alterações nos níveis de testosterona em mulheres. 
Cabe ressaltar as principais limitações do estudo, como o número reduzido de participantes e a não adição de grupo controle. No presente estudo não foi realizada a análise de marcadores de dano muscular como Creatina Quinase e nem coleta e análise sanguínea em outros momentos em diferentes minutos após o exercício, o que poderia indicar mudanças nas respostas agudas tardias dede testosterona e cortisol.

\section{CONSIDERAÇÕES FINAIS}

Portanto, observou-se a predominância de um estado catabólico após a realização de uma sessão de treinamento de força excêntrico com $110 \%$ da carga obtida no teste de $10 \mathrm{RM}$, o qual induziu respostas agudas com maiores níveis de cortisol e menores níveis de testosterona comparados aos níveis em repouso. É de grande importância a realização de futuras pesquisas visando analisar os níveis destes hormônios em um estado agudo tardio e também a análise destes de forma crônica. 


\section{Referências}

ARAÚJO, M. R. A influência do treinamento de força e do treinamento aeróbio sobre as concentrações hormonais de testosterona e cortisol. Motricidade, v. 4, n. 2, p. 67-75, 2008.

Badillo, Juan José Gonzáles.; Ayestarán, Esteban Gorostiaga; Fundamentos do Treinamento de Forca:Aplicação ao Alto Rendimento Desportivo-2 ed.- Porto Alegre : Artmed Editora ,2001.

BROWNLEE, K. K.; MOORE, A. W.; HACKNEY, A. C. Relationship between circulatingcortisol and testosterone: influence of physical exercise. Journal of sports science \& medicine, v. 4, n. 1, p. 76, 2005.

CADORE, Eduardo Lusa et al. Fatores relacionados com as respostas da testosterona e do cortisol ao treinamento de força. Revista brasileira de medicina do esporte. São Paulo. Vol. 14, n. 1 (jan./fev. 2008), p. 74-78, 2008.

CALIXTO, R. D. et al. Acute effects of movement velocity on blood lactate and growth hormone responses after eccentric bench press exercise in resistance-trained men.Biology of Sport, v. 31, n. 4, p. 289, 2014.

COLTINHO, H.; BRINCO, R. A.; DINIZ, S. H. Respostas hormonais da testosterona e cortisol depois de determinado protocolo de hipertrofia muscular. RBPFEXRevistaBrasileira de Prescrição e Fisiologia do Exercício, v. 1, n. 3, 2011.

FRANÇA, S. C. A. et al. Resposta divergente da testosterona e do cortisol séricos em atletas masculinos após uma corrida de maratona. Arq. bras. endocrinol. metab, v. 50, n. 6, p. 1082-1087, 2006.

GERLINGER-ROMERO, Frederico et al. Bases moleculares das ações da testosterona, hormônio do crescimento e igf-1 sobre a hipertrofia muscular esquelética e respostas ao treinamento de força. Revista Mackenzie de Educação Física e Esporte, v. 12, n. 2, 2014.

GUYTON, A. C. Fisiologia Humana, 6ª Edição, Ed.Guanabara, 1998.

KRAEMER, W. J.; RATAMESS, N. A. Hormonal responses and adaptations to resistance exercise and training. Sports Medicine, v. 35, n. 4, p. 339-361, 2005.

RAHIMI, R.; ROHANI, H.; EBRAHIMI, M. Efectos de los períodos de reposomuycortos em larelación de testosterona y cortisol durante ejercicios de alta resistenciaenhombres. Apunts: Medicina de l'esport, v. 46, n. 171, p. 145-149, 2011. 
ROIG, M. et al. The effects of eccentric versus concentric resistance training on muscle strength and mass in healthy adults: a systematic review with meta-analyses. British journalofsports medicine, 2008.

SÁ, M. J. P. M. et al. Efeitos do treino de força nos níveis de testosterona e cortisol. 2014.

SILVA JR, A. J. et al. Study of the behavior of cortisol, gh and insulin after a session of acute resisted exercise. Revista Brasileira de Medicina do Esporte, v. 20, n. 1, p. 21-25, 2014.

SIMMONS, P. S. et al. Increased proteolysis.An effect of increases in plasma cortisol within the physiologic range.Journal of Clinical Investigation, v. 73, n. 2, p. 412, 1984.

TEO, W.; NEWTON, M. J.; MCGUIGAN, M. R. Circadian rhythms in exercise performance: Implications for hormonal and muscular adaptation. Journal of sports science \& medicine, v. 10, n. 4, p. 600, 2011.

UCHIDA, M. C. et al. Alteração da relação testosterona: cortisol induzida pelo treinamento de força em mulheres. Revista Brasileira de Medicina do Esporte, $v$. 10, n. 3, p. 165-8, 2004.

UCHIDA, M. C. et al. Efeito de diferentes protocolos de treinamento de força sobre parâmetros morfofuncionais, hormonais e imunológicos. Revista Brasileira de Medicina do Esporte, v. 12, n. 1, p. 21- 26, 2006.

Viru A, Viru M. Assessingchanges in adaptivity for optimizing training strate- gies. In: Viru A, Viru M, editors. Biochemical monitoring of sport training. Champaign: HumanKinetics, 2001;193-220.

SMILIOS, ILIAS et al. Hormonal responses after various resistance exercise protocols. Medicine and science in sports and exercise, v. 35, n. 4, p. 644-654, 2003.

AHTIAINEN, Juha P. et al. Short vs. long rest period between the sets in hypertrophic resistance training: influence on muscle strength, size, and hormonal adaptations in trained men. The Journal of Strength \&

Conditioning Research, v. 19, n. 3, p. 572-582, 2005. 